\title{
Thioester-Containing Proteins in the Drosophila melanogaster Immune Response against the Pathogen Photorhabdus
}

\author{
Ioannis Eleftherianos*(D) and Upasana Sachar ${ }^{D}$ \\ Infection and Innate Immunity Lab, Department of Biological Sciences, The George Washington University, \\ Washington, DC 20052, USA; upasanashokal@gmail.com \\ * Correspondence: ioannise@gwu.edu; Tel.: +1-202-994-2367
}

Received: 26 December 2019; Accepted: 23 January 2020; Published: 28 January 2020

\begin{abstract}
The fruit fly Drosophila melanogaster forms a magnificent model for interpreting conserved host innate immune signaling and functional processes in response to microbial assaults. In the broad research field of host-microbe interactions, model hosts are used in conjunction with a variety of pathogenic microorganisms to disentangle host immune system activities and microbial pathogenicity strategies. The pathogen Photorhabdus is considered an established model for analyzing bacterial virulence and symbiosis due to its unique life cycle that extends between two invertebrate hosts: an insect and a parasitic nematode. In recent years, particular focus has been given to the mechanistic participation of the D. melanogaster thioester-containing proteins (TEPs) in the overall immune capacity of the fly upon response against the pathogen Photorhabdus alone or in combination with its specific nematode vector Heterorhabditis bacteriophora. The original role of certain TEPs in the insect innate immune machinery was linked to the antibacterial and antiparasite reaction of the mosquito malaria vector Anopheles gambiae; however, revamped interest in the immune competence of these molecules has recently emerged from the D. melanogaster-Photorhabdus infection system. Here, we review the latest findings on this topic with the expectation that such information will refine our understanding of the evolutionary immune role of TEPs in host immune surveillance.
\end{abstract}

Keywords: Drosophila melanogaster; thioester-containing proteins; Photorhabdus; Heterorhabditis; humoral immunity; cellular immunity; metabolism; infection

\section{Introduction}

Insects are excellent systems for analyzing the molecular and functional basis of host-pathogen interactions [1]. In particular, the fruit fly Drosophila melanogaster has played a crucial role in determining the signaling pathways and mechanisms they regulate in response to infection with a diverse range of microorganisms, including insect-specific pathogens as well as pathogens that infect humans. In the past several years, fine studies using genetic and genomic tools in D. melanogaster have uncovered evolutionarily conserved mechanisms of host defense [2-4]. These findings have significantly advanced the broad field of innate immunology and opened novel avenues of investigation into the identification and characterization of immune reactions directed against eukaryotic parasites [5]. Innate immune antimicrobial responses in D. melanogaster include systemic and tissue-specific humoral and cellular processes controlled by the Toll and Immune Deficiency (Imd) signaling pathways with the c-Jun-terminal kinase (Jnk) pathway and the Janus kinase (Jak)/signal transducer and activator of transcription (Stat) participating in complementary immune activities [6-9]. 
Entomopathogenic nematodes in the genus Heterorhabditis are widely used in crop protection practices for the management of a wide range of insect pests that mainly live in the soil. Their high efficiency is due to their ability to penetrate the insect cuticle and rapidly migrate in the host, their ability during infection to produce molecules that compromise the insect immune system, and their species-specific association with the Gram-negative bacteria Photorhabdus that synthesize and secrete a cocktail of virulence factors, which accelerate insect death by targeting mainly the gut and fat body as well as the hemocytes $[10,11]$. Because of these complex interactions involving two invertebrate animals and a microbe, entomopathogenic nematodes are also premier experimental tools for deciphering gene-for-gene interactions that define bacterial symbiosis and pathogenicity, nematode parasitism, and insect immunity [12]. The properties of this model have been recently adopted to dissect the insect host immune response against the nematode and their related bacteria when infected together or separately. These investigations have led to the determination of the genetic basis of host innate immune processes activated in response to the combined assault of the two pathogens or each one individually $[13,14]$.

Here, we review the most recent information on the association of thioester-containing proteins (TEPs) in the interaction between D. melanogaster and the Heterorhabditis symbionts Photorhabdus and the impact of these molecules on the host response to these entomopathogenic bacteria. TEPs are critical factors for modulating innate immunity in D. melanogaster [15]. Accumulating knowledge on host-parasite relationships will lead to the discovery of novel nematode-bacterial strategies for targeting specific host immune-related components as well as host defense systems designed to oppose deadly attacks by entomopathogens.

\section{Thioester-Containing Proteins in the Drosophila Humoral Response against Photorhabdus}

The humoral arm of the D. melanogaster host defense is mostly based on the activation of microbial recognition receptors that induce intracellular signals regulated by evolutionarily conserved pathways, a process that ultimately leads to the synthesis and secretion of antimicrobial peptides in the open circulatory system of the fly $[16,17]$. However, the connection between Tep gene activity and regulation of humoral immune activity in D. melanogaster responding to entomopathogenic bacteria and their nematode vectors forms a recently explored research area in the broad field of insect immunology.

Recently, a series of studies analyzed immune signaling variation in flies deficient for certain Tep genes in the context of infection with the potent insect pathogenic bacterium Photorhabdus [18]. Because Tep4 was upregulated in D. melanogaster adults in response to infection with either Photorhabdus luminescens or Photorhabdus asymbiotica bacteria and Tep4 loss-of-function mutant flies were resistant to these pathogens, the investigators estimated the transcriptional expression of readout genes in Photorhabdus-infected Tep4 mutant flies. The results demonstrated that two antimicrobial-peptide-encoding genes Defensin and Cecropin-A1 were highly upregulated in the Tep4 mutant flies in relation to the background controls when infected with either bacterial species, indicating the regulatory role of Tep4 in the Toll and Imd signaling of D. melanogaster to Photorhabdus. In a similar fashion, it was further shown that Tep6 mutants were resistant to both Photorhabdus species, while Tep 2 mutants were resistant to P. asymbiotica bacteria only, and that Tep 6 but not Tep 2 expression was required for the activation of Toll signaling, while Tep2 only modulated Imd signaling [19]. In addition, Jak/Stat activity against either Photorhabdus species when Tep2 is normally expressed and both Tep 2 and Tep6 are needed for proper Jnk signaling in flies responding specifically to P. asymbiotica (Figure 1). 


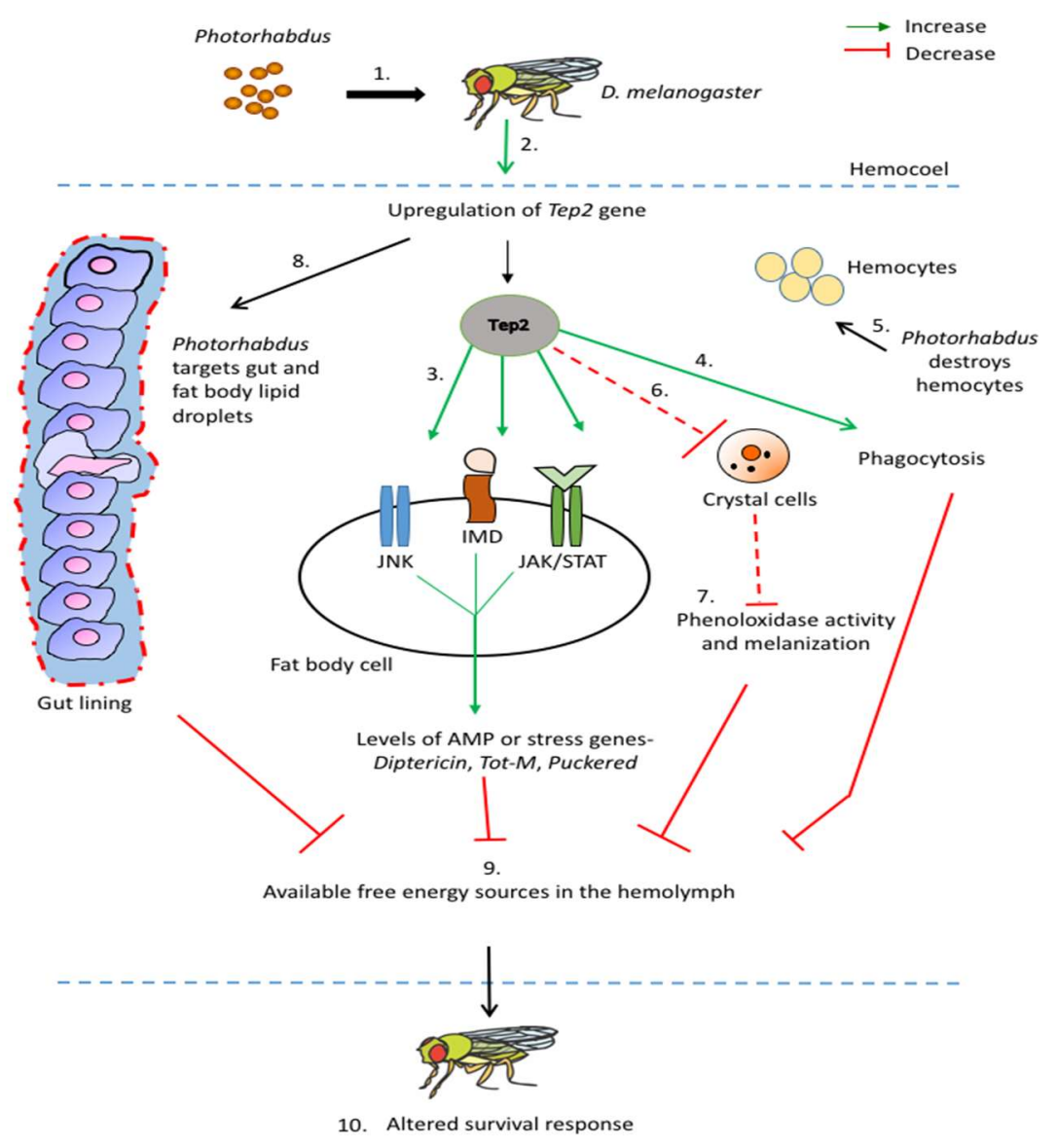

Figure 1. Model for the participation of TEP2 in the humoral and cellular immune response of Drosophila melanogaster wild-type adult flies against Photorhabdus entomopathogenic bacteria. 1. Photorhabdus enters the hemolymph of the flies. 2. Tep2 is upregulated in fly tissues such as fat body and hemocytes after Photorhabdus injection. 3. Upregulation of Tep2 results in the activation of Immune Deficiency (Imd), Janus kinase (Jak)/signal transducer and activator of transcription (Stat), and c-Jun-terminal kinase (Jnk) pathways leading to upregulation of antimicrobial peptide (AMP) and stress-related genes such as Diptericin, Turandot-M (Tot-M), and Puckered. 4. Upregulation of Tep2 increases phagocytosis in response to Photorhabdus infection. 5. Photorhabdus targets and kills hemocytes (specifically plasmatocytes). 6. Upregulation of Tep2 results in the appearance of fewer crystal cells in the hemolymph after Photorhabdus infection. 7. Reduced number of crystal cells leads to decreased phenoloxidase activity and melanization response. 8. Photorhabdus targets the gut (shown as red dashed border) and fat body lipid droplets promoting increased pathogen burden in the fly and consequently leading to increased cell death (activation of caspase Dronc). 9. The fly uses available energy sources (such as triglycerides and trehalose) to combat Photorhabdus infection. 10. The interaction between the Drosophila immune system activated through Tep2 and Photorhabdus pathogenicity strategies results in altered survival of the fly. Solid red lines denote inhibition and dashed red lines denote decrease.

\section{Thioester-Containing Proteins in the Drosophila Cellular Response against Photorhabdus}

The cellular part of the innate immune response in D. melanogaster operates together with humoral factors to eliminate microbial intruders. Cellular immunity in D. melanogaster larvae and adult flies is controlled by the different types of hemocytes, which specialize in various immune activities that mainly include the detection, phagocytosis, and encapsulation of pathogens [20,21].

To identify the effect of TEP molecules on the D. melanogaster antibacterial cell-mediated immunity, recent works have focused on estimating the activity of cellular responses in flies with compromised Tep gene expression upon infection with potent entomopathogenic Photorhabdus bacteria. It was found that the presence of functional hemocytes in adult D. melanogaster is essential for the expression of 
Tep2 and Tep 6 during infection with P. luminescens or P. asymbiotica bacteria, as functional inactivation of hemocytes in flies leads to decreased expression of these two genes in the presence of either pathogen [19]. Tep 2 and Tep 6 gene activity in D. melanogaster also impacts hemocyte activation and viability against Photorhabdus because total hemocyte numbers are altered in flies with mutations in either Tep 2 or Tep 6 , and these mutants contain a larger proportion of live hemocytes than control individuals when the pathogenic bacteria are present in the hemolymph (Figure 1). These changes are accompanied by modification in phagocytosis activity in the Tep mutant flies, as indicated by their reduced ability to engulf Escherichia coli inactive opsonized bioparticles and the differential expression of the phagocytic receptor Eater in response to Photorhabdus challenge [19]. In these experiments, E. coli particles were used because Photorhabdus pathogens are able to interfere with insect phagocytosis [14]. In another more detailed work, D. melanogaster TEP4 was also assigned a significant role in the regulation of cellular immune mechanisms against P. luminescens or P. asymbiotica bacteria [22]. Specifically, in flies compromised by these pathogenic bacteria, the presence of functional hemocytes is critical for the transcriptional expression of Tep4 at normal levels in wild-type flies as well as for the survival of Tep4 loss-of-function mutants. In addition, interfering with the expression of Tep4 in adult flies reduces total hemocyte numbers in the hemolymph, an effect that is accompanied by simultaneous increase in the proportion of crystal cells and changes in phagocytic ability against Photorhabdus.

The phenoloxidase and melanization reactions in D. melanogaster bridge the humoral and cellular arms of the innate antimicrobial immune response [23,24]. In a curious way, certain TEPs are also capable of modulating these two responses in the fly upon infection with entomopathogenic bacteria. In the absence of Tep 2 or Tep4, but not Tep6, both phenoloxidase activity in the hemolymph and melanization intensity at the injection site increase substantially in the mutants when responding to Photorhabdus infection (Figure 1) [18,19].

\section{Thioester-Containing Proteins and Drosophila Metabolism during Photorhabdus Infection}

An appealing question in insect immunology is the determination of the host factors that link immunity and metabolic function in the context of microbial infection. The close relationship between immune regulation and metabolic homeostasis in health and disease is well established [25-28], and evidence on the host signaling pathways and their molecules linking these important biological processes has recently started to emerge due to studies in the D. melanogaster model [29-31]. Because certain TEPs in D. melanogaster were shown to play a regulatory role by inducing humoral and cellular immune activities against Photorhabdus pathogens [18,19,22], these molecules also form a reliable indicator for their potential multipurpose involvement in linking host immunity and metabolism in the presence of pathogenic bacteria. Of note, injection of P. luminescens into the hemocoel of background wild-type flies upregulates both Tep2 and Tep4 in the fat body as well as Tep4 in the gut, whereas injection of P. asymbiotica increases the expression of Tep4 in the fat body only (Figure 1) [32]. Although the tissue-specific expression pattern of Tep genes in D. melanogaster varies upon infection with different bacterial pathogens [33], these results demonstrate that certain Tep genes are expressed in the two metabolic tissues of the fly - fat body and gut-in response to Photorhabdus systemic infection.

To address these issues, a recent study monitored the metabolic activity in D. melanogaster TEP-deficient flies during response to infection by two Photorhabdus species as well as by a nonpathogenic strain of E. coli used as control and estimated the amounts of different metabolites including carbohydrates and lipids [32]. Results from these experiments first revealed that trehalose levels are drastically affected in flies with compromised Tep2, regardless of whether they are infected with pathogenic Photorhabdus bacteria or nonpathogenic E. coli. Examination of glycogen levels similarly indicated that Tep4 gene activity is critical for the utilization of glycogen during the course of pathogenic or nonpathogenic challenge. Interestingly, glucose levels in infected D. melanogaster flies are differentially affected depending on the impairment of distinct Tep genes. More precisely, infection of Tep2 mutants with P. asymbiotica, but not P. luminescens, increases free glucose levels, while infection of Tep4-defective adults with either P. asymbiotica pathogens or nonpathogenic E. coli boosts glucose 
concentration in the infected flies. Also, both Tep2 and Tep4 gene activities in D. melanogaster adults seem to interfere with the regulation of triglyceride levels following Photorhabdus inoculation, since suppression of either Tep 2 or Tep 4 gene expression elevates the amount of triglycerides upon infection of the Tep mutant flies with either P. luminescens or P. asymbiotica compared with controls. Similarly, examination of fat body morphology in infected and uninfected D. melanogaster shows that Tep2- and Tep4-inactivated flies contain bigger-sized lipid droplets than infected wild-type individuals, an effect that is observable only during infection with pathogenic Photorhabdus bacteria but not in uninfected flies or those injected with E. coli. These results indicate that the absence of TEP2 or TEP4 may facilitate metabolic activity upon Photorhabdus infection. Overall, these findings have uncovered a previously unknown correlation between Tep gene expression and metabolic activity in D. melanogaster wild-type flies responding to the pathogen Photorhabdus. Future work is expected to resolve the exact involvement of TEP molecules in the D. melanogaster immunometabolic response to Photorhabdus infection.

\section{Thioester-Containing Protein Gene Expression in Drosophila and Stress and Inflammation Response to Photorhabdus}

Variations in insect survival and pathogen load often correlate with changes in host innate immune response, which is often difficult to separate from stress or inflammatory responses to a given microbial challenge [34,35]. A recent study expanded on this topic by examining the contribution of Tep gene activity to the stress and inflammation response to infection by Photorhabdus bacteria [32]. Because the nitrite level (a by-product of nitric oxide synthesis) is an indicator for stress response in the fly [36], the authors estimated nitrite amounts produced in D. melanogaster adults compromised for either Tep2 or Tep 4 in the context of infection with either P. luminescens or P. asymbiotica. The results from these experiments indicated that inactivation of either Tep gene leads to higher concentration of nitrite in the mutant flies infected with these pathogens. This effect is particularly pronounced during the late phase of the infection, which coincides with the spread of the bacteria in the fly hemocoel and colonization of the gut and fat body tissues [37]. In addition, Tep 2 and Tep 4 loss-of-function mutant flies injected with Photorhabdus bacteria exhibited lower expression of the caspase-encoding Dronc, which pointed towards decreased levels of apoptosis [38]. These findings were confirmed at the tissue level by detecting lower levels of protein expression of death caspase-1 in the midgut of Tep 2 and Tep4 Photorhabdus-infected flies [39]. The latter study provides evidence for the role of Tep gene activity in modulating cell death during infection with Photorhabdus pathogenic bacteria. Whether this effect is due to the higher numbers of Photorhabdus in the infected tissues due to the absence of apoptosis or the decreased release of the bacteria from the infected host cells that in turn might reduce the capacity of the pathogens to spread in the Tep mutant flies is currently unknown and therefore is worth investigating in future studies.

\section{Role of Thioester-Containing Proteins in the Drosophila Antinematode Response}

Recent transcriptomic studies in D. melanogaster have implicated the role of Tep genes in the interaction of parasitic nematode infection with the adult fly and larval innate immune response. Microarray analysis of the differentially regulated genes in $D$. melanogaster larvae responding to symbiotic Heterorhabditis bacteriophora identified Tep 2 among the 100 most enriched genes as well as Tep1, and the C 3 homolog Tep3 and Tep4 were also induced by infection with these nematode parasites [40]. Analysis of the survival response of Tep loss-of-function mutant larvae upon challenge with $H$. bacteriophora symbiotic nematodes revealed increased sensitivity of individuals with defective Tep3 only or when together with Tep2, while deficiencies in Тep 2 or Tep 4 alone failed to differentiate larval survival ability compared to background controls. Although further functional analysis was not attempted in this study, the obtained findings indicate that certain TEP molecules play an as-yet unidentified direct or indirect role in regulating the antinematode immune reaction [40]. Similar to the research with Photorhabdus bacteria, the functional basis of this reaction might be linked to the capacity of TEPs (TEP3 in this case and probably TEP2) to enhance the activity of humoral immune components 
or influence the stimulation and efficacy of immune cells to recognize and entrap parasitic nematodes. Interestingly, a subsequent RNA-sequencing study involving exposure of $D$. melanogaster adults with either symbiotic (carrying P. luminescens bacteria) or axenic (lacking P. luminescens) nematodes of $H$. bacteriophora showed that either type of infection results in consistent downregulation of Tep 1 at early and late times after the introduction of the pathogens to the flies, while Tep 1 and Tep 2 together with Tep 4 are highly upregulated by the parasites as well as by injection of $P$. luminescens bacteria alone throughout the infection [41]. Similar to the work with H. bacteriophora, infection of D. melanogaster larvae with the entomopathogenic nematodes Steinernema carpocapsae containing their symbiotic Xenorhabdus nematophila bacteria leads to high levels of induction for Tep 1 and Tep2, which remains steady during the infection [42]. Therefore, the challenge awaiting in the future involves the identification and mechanistic characterization of each of these transcriptomically detected TEPs in D. melanogaster, or more broadly, insect antinematode innate immune action.

\section{Conclusions}

The insect immune system includes extracellular and intracellular signaling pathways, which provide an efficient and rapid response against a variety of microbial pathogens that constantly employ novel tactics to undermine the host defense. Insect TEPs belong to a selective group of molecules with evolutionarily conserved function to first recognize the presence of microbial intruders and subsequently activate diverse immune mechanisms that restrict microbial growth and facilitate microbial elimination from the host [43]. In D. melanogaster, recent research employing the model pathogenic bacterium Photorhabdus and its parasitic nematode vector Heterorhabditis has assigned a putative role as immune regulators to TEP2, TEP4, and TEP6, which are markedly induced in response to Photorhabdus and evidently possess the capacity to control humoral and cellular reactions during infection with the mutualistic bacteria alone or the nematode-bacteria complexes. Changes in TEP transcriptional activity in $D$. melanogaster adult flies substantially modify the potency of immune activities including variation in innate immune signaling and therefore expression of antimicrobial peptide and stress-related genes as well as a decline in phenoloxidase and melanization responses. These drastic changes in turn alter the survival ability of the flies upon challenge with the pathogens. These findings in the D. melanogaster model have transformed our current knowledge of the existence of factors that are able to adjust the insect host innate immune system to oppose specific microbial invaders. Future focus should be given to analyzing the tissue-specific expression of Tep genes in D. melanogaster responding to nematode infection and determine the functional involvement of each TEP molecule in the fly antinematode defense. Such research will aim at refining further the details of these mechanisms with the prospect that the outcome will lead to more efficient strategies for the management of deleterious crop pests and vectors of deadly diseases.

Author Contributions: I.E.; writing—original draft preparation, U.S.; writing-review, editing, and preparation of the figure. All authors have read and agreed to the published version of the manuscript.

Funding: Research on Drosophila thioester-containing proteins was supported by a start-up fund from the Columbian College of Arts and Sciences at GWU to I.E., and the Harlan Summer Fellowships from the Department of Biological Sciences at GWU and a scholarship from the Cosmos Club Foundation (Washington, D.C.) to U.S.

Acknowledgments: We thank members of the Department of Biological Sciences for critical reading of the manuscript.

Conflicts of Interest: The authors declare no conflict of interest. The funders had no role in the design of the study; in the collection, analyses, or interpretation of data; in the writing of the manuscript; or in the decision to publish the results.

\section{References}

1. Sackton, T.B. Comparative genomics and transcriptomics of host-pathogen interactions in insects: Evolutionary insights and future directions. Curr. Opin. Insect Sci. 2019, 31, 106-113. [CrossRef] [PubMed] 
2. Buchon, N.; Silverman, N.; Cherry, S. Immunity in Drosophila melanogaster-From microbial recognition to whole-organism physiology. Nat. Rev. Immunol. 2014, 14, 796-810. [CrossRef] [PubMed]

3. Gold, K.S.; Brückner, K. Macrophages and cellular immunity in Drosophila melanogaster. Semin. Immunol. 2015, 27, 357-368. [CrossRef]

4. Parsons, B.; Foley, E. Cellular immune defenses of Drosophila melanogaster. Dev. Comp. Immunol. 2016, 58, 95-101. [CrossRef]

5. Keebaugh, E.S.; Schlenke, T.A. Insights from natural host-parasite interactions: The Drosophila model. Dev. Comp. Immunol. 2014, 42, 111-123. [CrossRef]

6. Lindsay, S.A.; Wasserman, S.A. Conventional and non-conventional Drosophila Toll signaling. Dev. Comp. Immunol. 2014, 42, 16-24. [CrossRef]

7. Zhai, Z.; Huang, X.; Yin, Y. Beyond immunity: The Imd pathway as a coordinator of host defense, organismal physiology and behavior. Dev. Comp. Immunol. 2018, 83, 51-59. [CrossRef]

8. Myllymäki, H.; Rämet, M. JAK/STAT pathway in Drosophila immunity. Scand. J. Immunol. 2014, 79, 377-385. [CrossRef]

9. Delaney, J.R.; Stöven, S.; Uvell, H.; Anderson, K.V.; Engström, Y.; Mlodzik, M. Cooperative control of Drosophila immune responses by the JNK and NF-kappaB signaling pathways. EMBO J. 2006, 25, 3068-3077. [CrossRef]

10. Brivio, M.F.; Mastore, M. Nematobacterial Complexes and Insect Hosts: Different Weapons for the Same War. Insects 2018, 9, 117. [CrossRef]

11. Cooper, D.; Eleftherianos, I. Parasitic Nematode Immunomodulatory Strategies: Recent Advances and Perspectives. Pathogens 2016, 5, 58. [CrossRef] [PubMed]

12. Shi, Y.M.; Bode, H.B. Chemical language and warfare of bacterial natural products in bacteria-nematode-insect interactions. Nat. Prod. Rep. 2018, 35, 309-335. [CrossRef] [PubMed]

13. Castillo, J.C.; Reynolds, S.E.; Eleftherianos, I. Insect immune responses to nematode parasites. Trends Parasitol. 2011, 27, 537-547. [CrossRef] [PubMed]

14. Eleftherianos, I.; ffrench-Constant, R.H.; Clarke, D.J.; Dowling, A.J.; Reynolds, S.E. Dissecting the immune response to the entomopathogen Photorhabdus. Trends Microbiol. 2010, 18, 552-560. [CrossRef] [PubMed]

15. Lu, Y.; Su, F.; Li, Q.; Zhang, J.; Li, Y.; Tang, T.; Hu, Q.; Yu, X.Q. Pattern recognition receptors in Drosophila immune responses. Dev. Comp. Immunol. 2020, 102, 103468. [CrossRef]

16. Ganesan, S.; Aggarwal, K.; Paquette, N.; Silverman, N. NF-kappaB/Rel proteins and the humoral immune responses of Drosophila melanogaster. Curr. Top. Microbiol. Immunol. 2011, 349, 25-60.

17. Imler, J.L.; Bulet, P. Antimicrobial peptides in Drosophila: Structures, activities and gene regulation. Chem. Immunol. Allergy 2005, 86, 1-21.

18. Shokal, U.; Eleftherianos, I. Thioester-Containing Protein-4 Regulates the Drosophila Immune Signaling and Function against the Pathogen Photorhabdus. J. Innate Immun. 2017, 9, 83-93. [CrossRef]

19. Shokal, U.; Kopydlowski, H.; Eleftherianos, I. The distinct function of Tep2 and Tep6 in the immune defense of Drosophila melanogaster against the pathogen Photorhabdus. Virulence 2017, 8, 1668-1682. [CrossRef]

20. Wood, W.; Martin, P. Macrophage Functions in Tissue Patterning and Disease: New Insights from the Fly. Dev. Cell 2017, 40, 221-233. [CrossRef]

21. Honti, V.; Csordás, G.; Kurucz, É.; Márkus, R.; Andó, I. The cell-mediated immunity of Drosophila melanogaster: Hemocyte lineages, immune compartments, microanatomy and regulation. Dev. Comp. Immunol. 2014, 42, 47-56. [CrossRef] [PubMed]

22. Shokal, U.; Eleftherianos, I. The Drosophila Thioester-Containing Protein-4 participates in the induction of the cellular immune response to the pathogen Photorhabdus. Dev. Comp. Immunol. 2017, 76, 200-208. [CrossRef] [PubMed]

23. Tang, H. Regulation and function of the melanization reaction in Drosophila. Fly 2009, 3, 105-111. [CrossRef] [PubMed]

24. Eleftherianos, I.; Revenis, C. Role and importance of phenoloxidase in insect hemostasis. J. Innate Immun. 2011, 3, 28-33. [CrossRef]

25. Ganeshan, K.; Chawla, A. Metabolic regulation of immune responses. Annu. Rev. Immunol. 2014, 32, 609-634. [CrossRef]

26. Vavricka, C.J.; Han, Q.; Mehere, P.; Ding, H.; Christensen, B.M.; Li, J. Tyrosine metabolic enzymes from insects and mammals: a comparative perspective. Insect Sci. 2014, 21, 13-19. [CrossRef] 
27. Brestoff, J.R.; Artis, D. Immune regulation of metabolic homeostasis in health and disease. Cell 2015, 161, 146-160. [CrossRef]

28. Zmora, N.; Bashiardes, S.; Levy, M.; Elinav, E. The Role of the Immune System in Metabolic Health and Disease. Cell Metab. 2017, 25, 506-521. [CrossRef]

29. Dionne, M. Immune-metabolic interaction in Drosophila. Fly 2014, 8, 75-79. [CrossRef]

30. Lee, K.A.; Lee, W.J. Immune-metabolic interactions during systemic and enteric infection in Drosophila. Curr. Opin. Insect Sci. 2018, 29, 21-26. [CrossRef]

31. Galenza, A.; Foley, E. Immunometabolism: Insights from the Drosophila model. Dev. Comp. Immunol. 2019, 94, 22-34. [CrossRef] [PubMed]

32. Shokal, U.; Kopydlowski, H.; Harsh, S.; Eleftherianos, I. Thioester-Containing Proteins 2 and 4 Affect the Metabolic Activity and Inflammation Response in Drosophila. Infect. Immun. 2018, 86, e00810-17. [CrossRef] [PubMed]

33. Bou Aoun, R.; Hetru, C.; Troxler, L.; Doucet, D.; Ferrandon, D.; Matt, N. Analysis of thioester-containing proteins during the innate immune response of Drosophila melanogaster. J. Innate Immun. 2011, 3, 52-64. [CrossRef] [PubMed]

34. Davies, S.A.; Overend, G.; Sebastian, S.; Cundall, M.; Cabrero, P.; Dow, J.A.; Terhzaz, S. Immune and stress response 'cross-talk' in the Drosophila Malpighian tubule. J. Insect Physiol. 2012, 58, 488-497. [CrossRef] [PubMed]

35. Asri, R.M.; Salim, E.; Nainu, F.; Hori, A.; Kuraishi, T. Sterile induction of innate immunity in Drosophila melanogaster. Front. Biosci. 2019, 24, 1390-1400.

36. Dow, J.A.; Romero, M.F. Drosophila provides rapid modeling of renal development, function and disease. Am. J. Physiol. Renal Physiol. 2010, 299, F1237-F1244. [CrossRef]

37. Nielsen-LeRoux, C.; Gaudriault, S.; Ramarao, N.; Lereclus, D.; Givaudan, A. How the insect pathogen bacteria Bacillus thuringiensis and Xenorhabdus/Photorhabdus occupy their hosts. Curr. Opin. Microbiol. 2012, 15, 220-231. [CrossRef]

38. Ming, M.; Obata, F.; Kuranaga, E.; Miura, M. Persephone/Spätzle pathogen sensors mediate the activation of Toll receptor signaling in response to endogenous danger signals in apoptosis-deficient Drosophila. J. Biol. Chem. 2014, 289, 7558-7568. [CrossRef]

39. Song, Z.; McCall, K.; Steller, H. DCP-1, a Drosophila cell death protease essential for development. Science 1997, 275, 536-540. [CrossRef]

40. Arefin, B.; Kucerova, L.; Dobes, P.; Markus, R.; Strnad, H.; Wang, Z.; Hyrsl, P.; Zurovec, M.; Theopold, U. Genome-wide transcriptional analysis of Drosophila larvae infected by entomopathogenic nematodes shows involvement of complement, recognition and extracellular matrix proteins. J. Innate Immun. 2014, 6, 192-204. [CrossRef]

41. Castillo, J.C.; Creasy, T.; Kumari, P.; Shetty, A.; Shokal, U.; Tallon, L.J.; Eleftherianos, I. Drosophila anti-nematode and antibacterial immune regulators revealed by RNA-seq. BMC Genom. 2015, 11, 519. [CrossRef] [PubMed]

42. Yadav, S.; Daugherty, S.; Shetty, A.C.; Eleftherianos, I. RNAseq analysis of the Drosophila response to the entomopathogenic nematode Steinernema. G3 (Bethesda) 2017, 7, 1955-1967. [CrossRef] [PubMed]

43. Shokal, U.; Eleftherianos, I. Evolution and Function of Thioester-Containing Proteins and the Complement System in the Innate Immune Response. Front. Immunol. 2017, 8, 759. [CrossRef] [PubMed]

(C) 2020 by the authors. Licensee MDPI, Basel, Switzerland. This article is an open access article distributed under the terms and conditions of the Creative Commons Attribution (CC BY) license (http://creativecommons.org/licenses/by/4.0/). 